\title{
ROTATING NEWTONIAN FLUIDS IN A NON-INERTIAL FRAME OF REFERENCE AMAN MEHTA ${ }^{1} \&$ DEEPAK CHOUDHARY ${ }^{2}$
}

${ }^{1}$ Student at University of Illinois at Urbana-Champaign

${ }^{2}$ Podar International School

Received: May 23, 2021; Accepted: Jun 13, 2021; Published: Sep 04, 2021; Paper Id.: IJPRDEC20214

\section{INTRODUCTION}

Stirring a liquid doesn't produce a flat surface but instead forms a dip in the middle and stirring it faster causes a greater depth. An adapted problem was proposed in the form of Newton's Bucket as Newton mentioned:i

“...the surface of the water will at first be flat, before it begins to recede from the centre and ascend up the sides of the bucket, forming itself into a concave figure, and the swifter the motion becomes, the higher will the water rise, till at last, it becomes relatively at rest in it. " ii

The lack of a defined mathematical relationship between "swifter the motion" and "higher will the water rise" inspired an adapted experiment to quantify this observation and determine the relation between the synonymous angular velocity of the rotating fluid and the parabolic depth formed upon rotation.

This topic is worthy of investigation because of its applications in our day-to-day lives. Rotating furnaces require a spinning liquid to create precise parabolic surfaces. These surfaces are utilised in fuel tanks of spinning rockets, solar cookers and centrifugal filters. ${ }^{\text {iii,iv }}$ Astronomical telescopes make use of rotating fluids as focal length adjustable parabolic mirrors. The determination of an appropriate angular velocity is necessary to create parabolic surfaces with a precise depth. ${ }^{\mathrm{v}}$ 


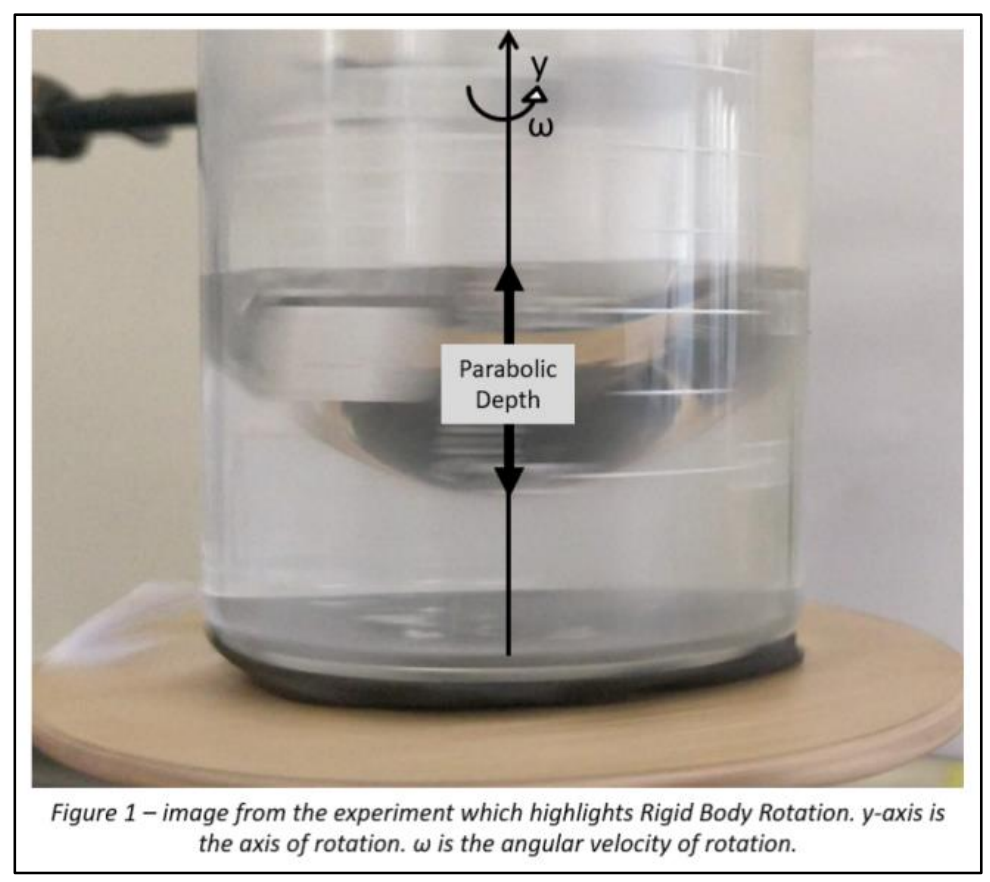

\section{Theory}

Figure 1 is an example of rigid body rotation, where a substance rotates around a fixed imaginary axis of rotation - y-axis consisting of all points inside the rotating body which remain stationary at a given angular velocity. ${ }^{\text {vi }}$ The entire fluid moves as a rigid body such that individual particles are not deforming and have no shear stresses. ${ }^{\text {vii }}$ The rotating fluid is stationary with respect to the rotating beaker and there is no relative motion between fluid particles; it appears that the water is at rest.

It is assumed that the fluid at the beaker wall is moving with the same angular velocity as the beaker and adhesion of liquid to the beaker is assumed large enough to neglect any slipping at the wall. However, in reality, there are frictional forces present between the liquid and the surface of the rotating vessel. There exists a boundary layer near the rotating vessel where the fluid velocity in that region is different from the rest of the fluid. viii

The derivation of the relationship between angular velocity of the fluid and its parabolic depth is considered in an inertial frame of reference since the consideration of a rotating (non-inertial) frame of reference along with hydrostatic force can produce "paradoxical results". ix

The following derivation assumes the consideration of Newtonian fluids only since Non-Newtonian fluids require a yield stress to overcome before rotational motion is initiated, which is not the case for Newtonian fluids. Additionally, resistance to free flowing motion (viscosity) is assumed to be sufficiently low. ${ }^{\mathrm{x}, \mathrm{xi}}$

The high number of assumptions in the model is a limitation of the experiment as not all of them may be met while conducting the experiment. 


\section{Derivation of the Relationship}
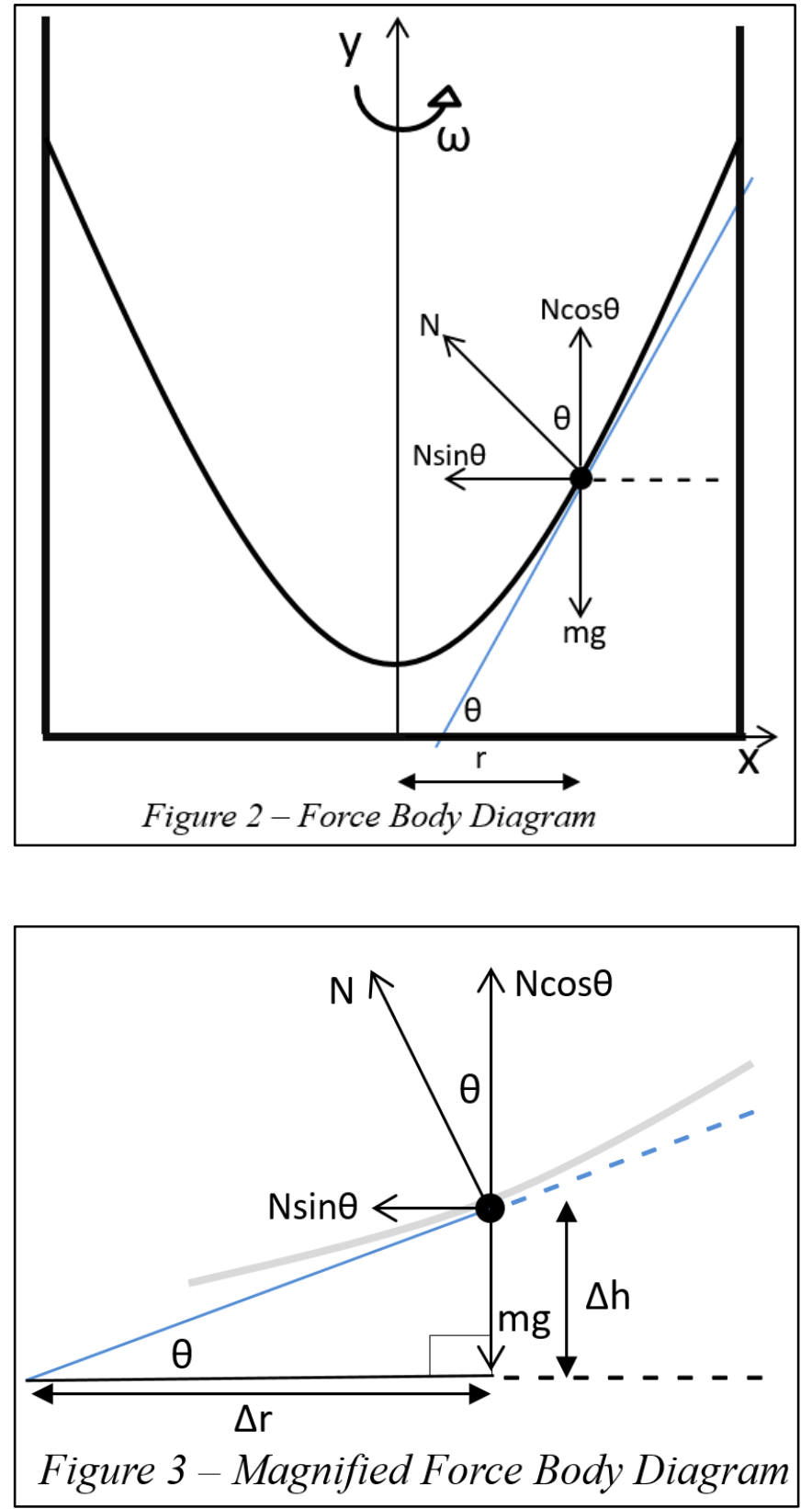

To determine forces on the rotating fluid, the forces a single droplet of water (denoted by the black circle) is considered:

The tangent in Figure 2 and 3 is used a reference line to mark angles.

Primarily, there are just two forces acting on the droplet of water in circular motion; first is the weight of the liquid acting downwards:

$$
F_{g}=m g
$$


$m$ is the mass of the water droplet and $g$ is the acceleration due to gravity.

The second force is the force created due to fluid pressure $-\mathrm{N}$ - which acts perpendicular to the tangent as seen in Figure 2 and 3. Inside the fluid, the pressure force is exerted isotropically - equally in all directions. However, this force becomes unbalanced at the parabolic surface since the force can only push the particles from inside the fluid. Hence, at the parabolic surface, the force created due to fluid pressure is directed outward and perpendicular to the surface. ${ }^{\mathrm{xii}}$

A centripetal force $\left(F_{c}\right)$ must act on the water droplet in circular motion with its centre on the y-axis. $F_{c}$ is not another force but is a component of $\mathrm{N}$ acting towards the centre:

$$
F_{c}=N \sin (\theta)
$$

$\mathrm{N} \sin (\Theta)$ is unbalanced (no opposing force) so it alone provides centripetal acceleration to keep the liquid moving in a circular path with angular velocity $\omega$. The liquid accelerates and $\mathrm{F}_{\mathrm{c}}$ is also given by:

$$
F_{c}=m \omega^{2} \Delta r
$$

$\Delta r$ is the distance from the centre of the circular path to the droplet of water.

Equating (2) and (3):

$N \sin (\theta)=m \omega^{2} r$

After the shape is formed, the weight of the particle, (1), and the cosine component of $\mathrm{N}, \mathrm{N} \cos \Theta$, are equal in magnitude but opposite in direction because the water droplet doesn't undergo any vertical displacement:

$$
N \cos (\theta)=m g
$$

Dividing (4) with (5):

$$
\begin{aligned}
& \frac{N \sin \theta}{N \cos \theta}=\frac{m \omega^{2} r}{m g} \\
& \tan (\theta)=\frac{\omega^{2} r}{g}
\end{aligned}
$$

The right angled triangle in Figure 3 suggests:

$$
\tan (\theta)=\frac{\Delta h}{\Delta r}=\frac{d h}{d r}
$$

Equating (7) and (8) gives the differential equation: 
$\frac{d h}{d r}=\frac{\omega^{2} r}{g}$

$$
d h=\frac{\omega^{2} r}{g} d r
$$

Integrating both sides:

$$
\int d h=\int \frac{\omega^{2} r}{g} d r
$$

$\mathbf{h}$ and $\mathbf{r}$ are integrated:

$$
\int d h=\frac{\omega^{2}}{g} \int r d r
$$

The general solution to this differential equation is:

$$
h=\frac{\omega^{2} r^{2}}{2 g}+h_{0}
$$

$h$ is the vertical displacement of an arbitrary point from the base of the container.

$h_{0}$ is the constant of integration and is the height from the base of the container to the minimum of the parabola formed.

In context of the research question, (10) is rearranged:

$$
h-h_{0}=\frac{\omega^{2} r^{2}}{2 g}
$$

The parabolic depth, $h_{d}$, is the vertical height difference between the minimum point of the parabola and the height of the surface at $r=r_{0}$ and is: ${ }^{x i i}$

$$
h_{d}=h-h_{0}
$$




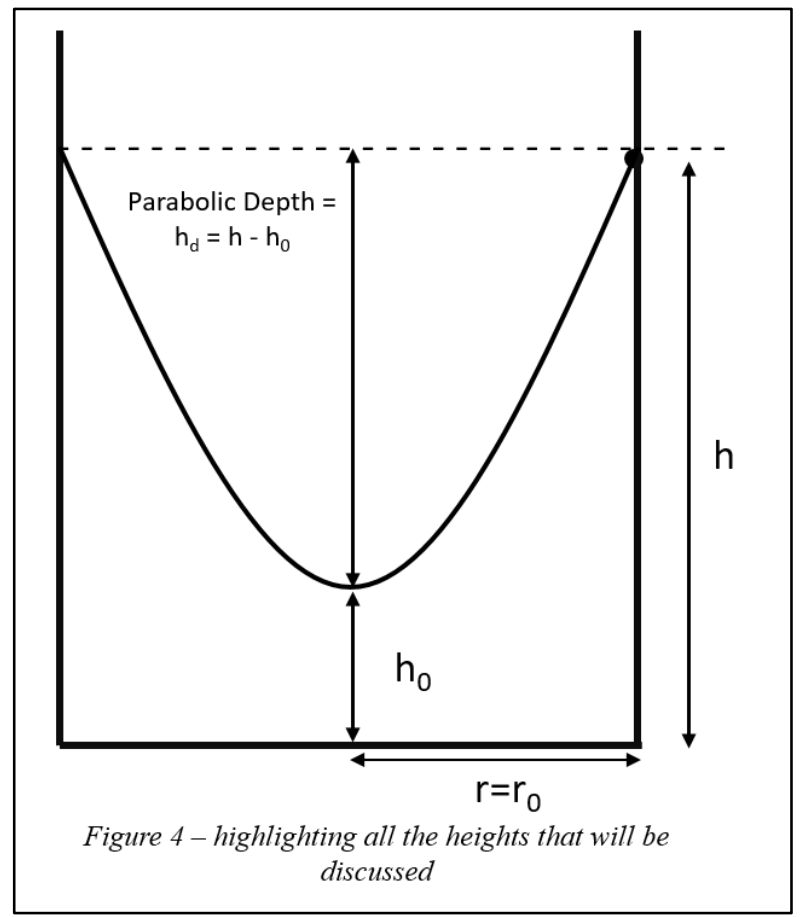

The different heights to be measured are:

$h$ is the vertical displacement for the point at $r=r_{0}$.

The conditions of this experiment were:

- $\quad \mathbf{h}_{\mathbf{d}} \leq \mathbf{h}$ and

- $\mathbf{h}_{\mathbf{0}} \geq \mathbf{0}$

These conditions imply that the minimum of the parabola must be above or at the surface of the beaker.

(12) is substituted in (11) and $r$ is substituted with $r_{0}$ - radius of the beaker:

$h_{d}=\frac{\omega^{2} r_{0}^{2}}{2 g}$

Relating the angular velocity $(\omega)$ to the parabolic depth $\left(\mathrm{h}_{\mathrm{d}}\right)$ :

$$
h_{d}=\frac{r_{0}^{2}}{2 g} \omega^{2}
$$

Replacing the constants ( $\mathrm{r}_{0}$ and $\mathrm{g}$ ):

$$
\begin{aligned}
& h_{d}=k \omega^{2} \\
& h_{d} \propto \omega^{2}
\end{aligned}
$$


The relationship to be investigated is shown in the equation of a parabola which relates angular velocity $(\omega)$ of the fluid to the parabolic depth $\left(h_{d}\right)$. It is predicted that plotting $h_{d}$ against $\omega^{2}$ should ideally give a straight line passing through the origin with a gradient given by the coefficient of $\omega^{2}$ from (14).

The reasoning behind the direct relationship between $\omega^{2}$ and $h_{d}$, from the Research Question, is understood through application of pressure:

$$
p=\frac{\rho \omega^{2} r_{0}{ }^{2}}{2}
$$

$\mathrm{p}$ is pressure on fluid particles and $\rho$ is the density of the fluid. ${ }^{\mathrm{xiv}, \mathrm{xv}, \mathrm{xvi}}$

This equation also demonstrates the parabolic nature of the curve. It shows that the pressure for points at the same distance, $r$, away from the axis of rotation, is the same. Thereby, the fluid rotating with higher angular velocity will have a higher pressure than the fluid rotating at a lower angular velocity ( $\Delta \mathrm{p}$ is positive).

Now, the equation above is re-arranged in terms of the variables defined to relate the fluid pressure to the parabolic depth: ${ }^{\text {vii }}$

$$
\begin{aligned}
& p=\rho g \times \frac{\omega^{2} r_{0}{ }^{2}}{2 g} \\
& p=\rho g h_{d}
\end{aligned}
$$

Since $\mathrm{g}$ is a constant and the density of the liquid remains same:

$$
p \propto h_{d}
$$

The pressure exerted on fluid particles is directly proportional to the parabolic depth. Thereby, (17) showed that the increasing angular velocity increases the pressure exerted on the fluid particles which causes a greater parabolic depth $\left(\mathrm{h}_{\mathrm{d}}\right)$, seen in (18).

\section{Experiment}

The aim of the experiment was to change the angular velocity $(\omega)$ of the fluid (which was done by changing the input voltage to the motor) and determine the parabolic depth $\left(h_{d}\right)$ measured by fixing a ruler next to rotating beaker.

Controlled variables are present to ensure that the angular velocity is the only factor which changes parabolic depth. These included: radius ( $r$ ) of the beaker, volume of water used, the time after which the height levels were measured, and the general setup. 


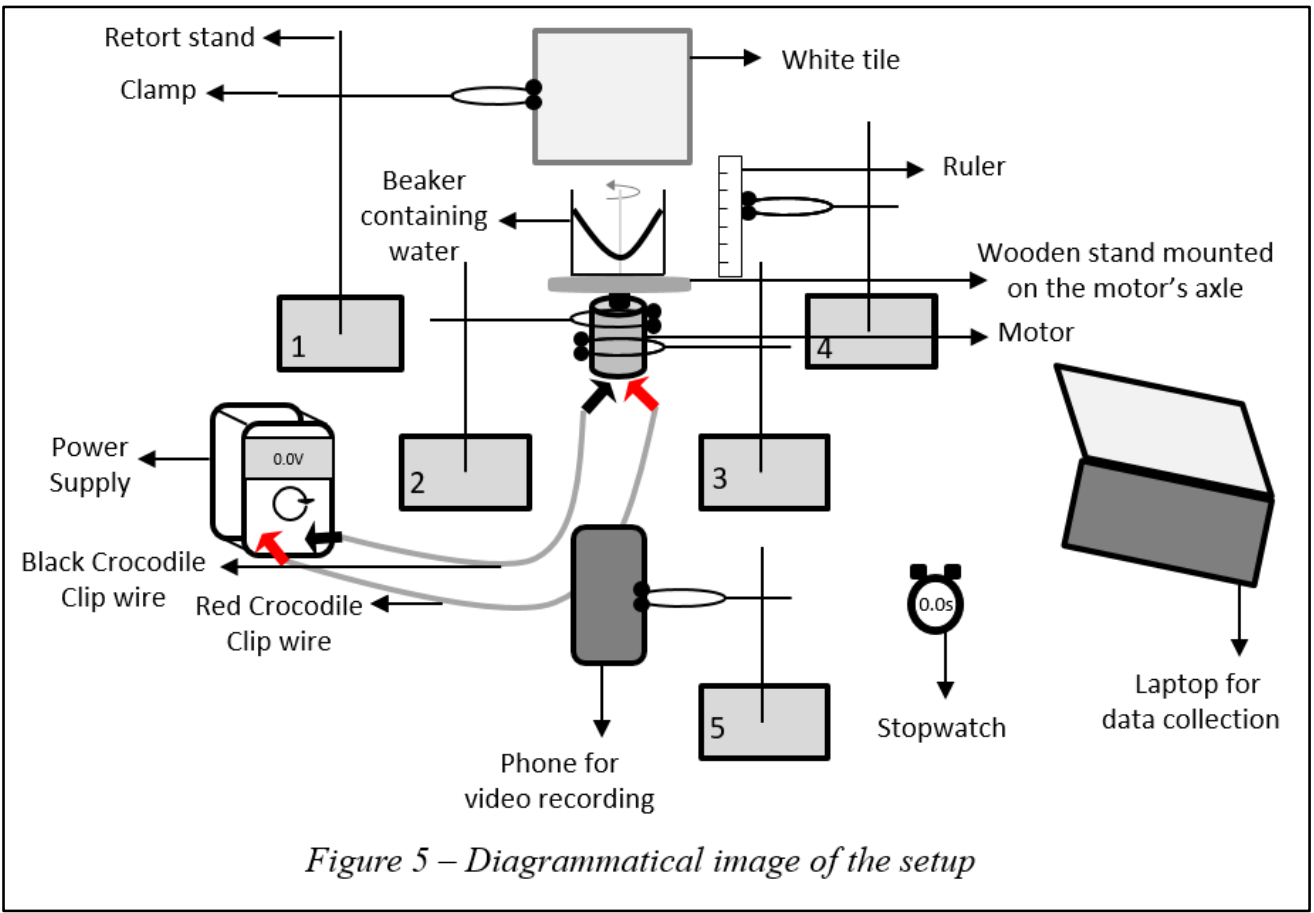

The diagram of the setup is seen in figure 5 .

The basic choice of materials from the school laboratory ensures the experiment can be repeated with ease.

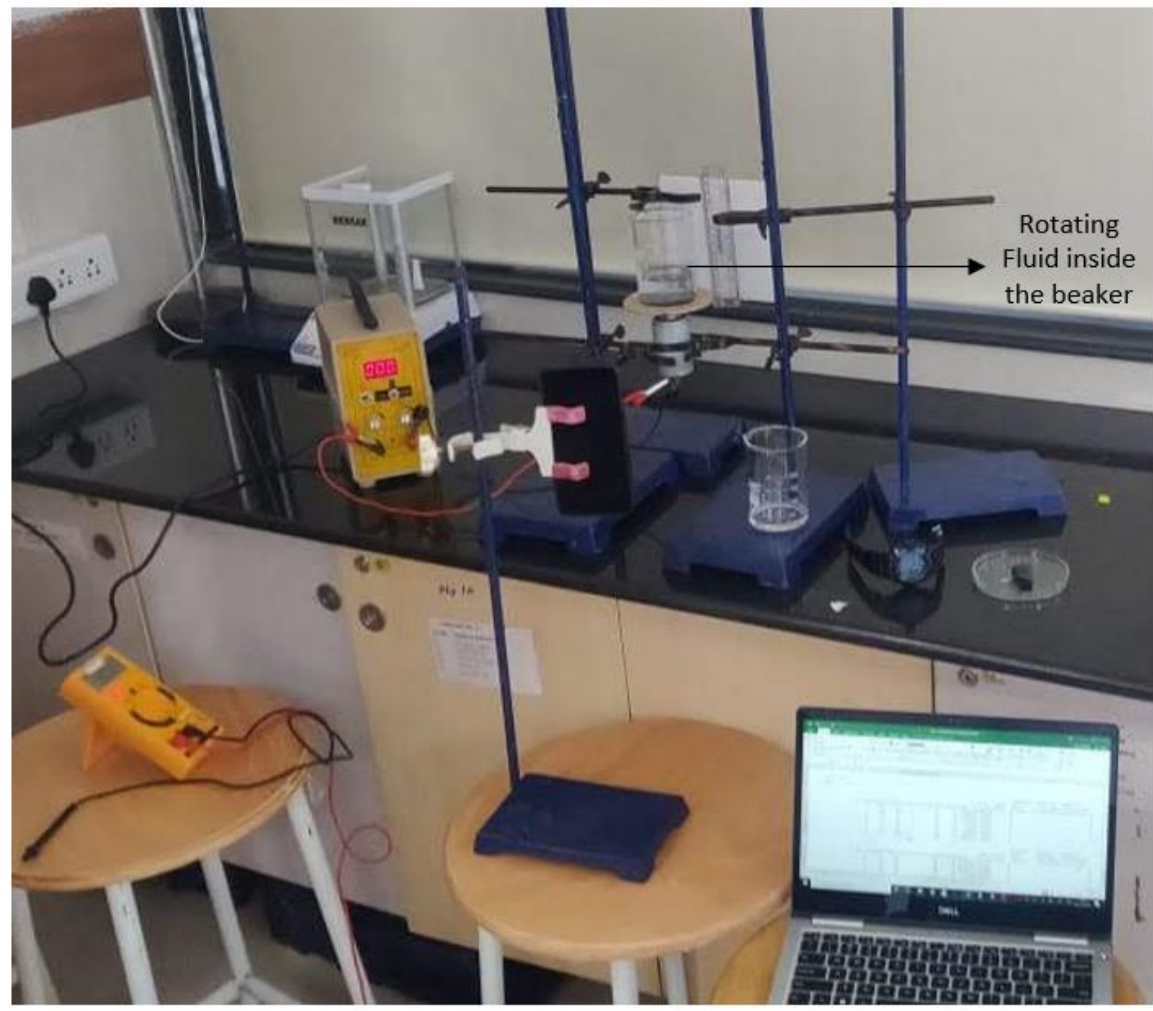

Figure 6 - image of the actual setup 
The actual setup is seen in figure 6.

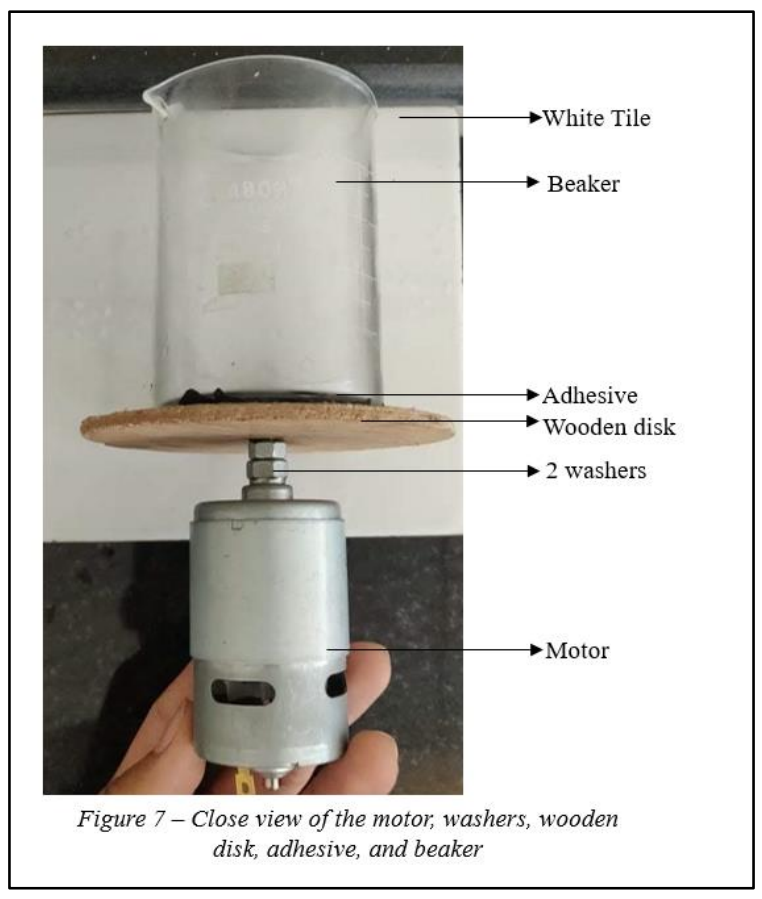

\section{Apparatus List}

5 retort stands with 5 clamps - to fix apparatus as seen in figure 6 . Circular wooden disk (radius $5.3 \mathrm{~cm} \pm 0.1 \mathrm{~cm}$ ) as the base of the beaker attached to it using an adhesive. 2 washers were used to fill the gap of the axle. Beaker containing 100 $\mathrm{ml}$ of water. Power supply used to alter the voltage supplied to the motor which changes the angular velocity. Stopwatch used while calculating angular velocity of the rotating beaker. $( \pm 0.01 \mathrm{~s})$. Motor - to rotate the wooden disk and the connected beaker. A close up view of the rotating beaker is shown in figure 7.

\section{Procedure}

The range of independent variables had to be decided which followed a criterion. The lowest angular velocity $\left(8.2 \mathrm{rad} \mathrm{s}^{-1}\right)$ should produce a parabola with non-negligible depth such that measurement is possible. The highest angular velocity (33.3 $\mathrm{rad} \mathrm{s}^{-1}$ ) should produce a complete parabola such that its minimum is above or at the base of the beaker. The other angular velocities must be between these limits and its overall range (in $\mathrm{rad} \mathrm{s}^{-1}$ ) is: 8.2, 11.9, 17.6, 22.4, 30.2, 33.3, and 39.6. However, one selected angular velocity (of $39.6 \mathrm{rad} \mathrm{s}^{-1}$ whose data was discounted) was above the upper limit to evaluate its parabolic shape.

Before determining the angular velocity, the time period was calculated using:

Time Period $=T=\frac{10 \text { seconds }}{\text { Revolutions in } 10 \text { seconds }}$

$\omega$ was calculated:

$\omega=\frac{2 \pi}{T}$

Here, it was earlier assumed that the angular velocity of the rotating beaker is the same as that of the rotating 
water. However, deviations from this assumption limit the accurate determination of the angular velocity values.

The voltage was first set such that the angular velocity was $8.2 \mathrm{rad} \mathrm{s}^{-1}$. Time of 1 minute was given for the water to come at rest with respect to the rotating beaker. The heights ( $h_{0}$ and $h$ from figure 4$)$ were measured and the parabolic depth $\left(h_{d}\right)$ was calculated.

\begin{tabular}{|c|c|c|c|c|c|c|c|c|c|c|c|c|c|c|c|c|}
\hline & & & \multicolumn{3}{|c|}{$\begin{array}{c}\text { Revolutions in 10 } \\
\text { seconds }\end{array}$} & \multicolumn{3}{c|}{$\mathrm{h}_{0}(\mathrm{~cm}) \pm 0.05 \mathrm{~cm}$} & \multicolumn{3}{c|}{$\mathrm{h}(\mathrm{cm}) \pm 0.05 \mathrm{~cm}$} \\
\hline Sr No. & Voltage (V) & $\begin{array}{c}\text { Time (s) } \pm \\
0.01 \mathrm{~s}\end{array}$ & $\mathrm{R} 1$ & $\mathrm{R} 2$ & $\mathrm{R} 3$ & $\begin{array}{c}\text { Height at rest } \\
(\mathrm{cm}) \pm 0.05 \mathrm{~cm}\end{array}$ & $\mathrm{~h}_{0} 1$ & $\mathrm{~h}_{0} 2$ & $\mathrm{~h}_{0} 3$ & $\mathrm{~h}_{0} 4$ & $\mathrm{~h}_{0} 5$ & $\mathrm{~h} 1$ & $\mathrm{~h} 2$ & $\mathrm{~h} 3$ & $\mathrm{~h} 4$ & $\mathrm{~h} 5$ \\
\hline 1 & 0.5 & 10.00 & 13 & 13 & 13 & 3.0 & 2.7 & 2.6 & 2.7 & 2.7 & 2.6 & 3.3 & 3.3 & 3.2 & 3.2 & 3.4 \\
\hline 2 & 0.6 & 10.00 & 19 & 21 & 19 & 3.0 & 2.6 & 2.5 & 2.5 & 2.6 & 2.6 & 3.5 & 3.4 & 3.3 & 3.6 & 3.5 \\
\hline 3 & 0.7 & 10.00 & 27 & 29 & 28 & 3.0 & 2.3 & 2.3 & 2.2 & 2.2 & 2.4 & 3.9 & 3.9 & 3.8 & 3.7 & 3.9 \\
\hline 4 & 0.9 & 10.00 & 36 & 36 & 35 & 3.0 & 1.6 & 1.7 & 1.6 & 1.6 & 1.5 & 4.4 & 4.5 & 4.4 & 4.4 & 4.3 \\
\hline 5 & 1.0 & 10.00 & 48 & 48 & 49 & 3.0 & 0.6 & 0.6 & 1.1 & 1.2 & 1.1 & 5.1 & 4.9 & 5.6 & 5.5 & 5.4 \\
\hline 6 & 1.1 & 10.00 & 53 & 54 & 52 & 3.0 & 0.0 & 0.1 & 0.1 & 0.0 & 0.0 & 6.1 & 6.3 & 6.3 & 6.2 & 6.1 \\
\hline 7 & 1.2 & 10.00 & 63 & 61 & 65 & 3.0 & N/A & N/A & N/A $/$ N/A & N/A & 7.1 & 7.0 & 6.7 & 7.2 & 7.0 \\
\hline
\end{tabular}

Table 1 - Raw Data

\section{Data Collection}

\begin{tabular}{|c|c|c|c|c|c|c|c|c|c|c|c|c|}
\hline \multirow[b]{2}{*}{ Sr No. } & \multirow[b]{2}{*}{$\begin{array}{l}\text { Time Period } \\
(\mathrm{s}) \pm 0.01 \mathrm{~s}\end{array}$} & \multirow[b]{2}{*}{$\begin{array}{c}\text { angular } \\
\text { velocity } \omega \\
\left(\mathrm{rad} \mathrm{s}^{-1}\right) \pm \\
0.01 \mathrm{rad} \mathrm{s}^{-1}\end{array}$} & \multirow[b]{2}{*}{$\begin{array}{c}\text { Error in } \omega \\
\left(\mathrm{rad} \mathrm{s}^{-1}\right)\end{array}$} & \multirow[b]{2}{*}{$\begin{array}{c}\omega^{2} \\
\left(\operatorname{rad}^{2} \mathrm{~s}^{-2}\right)\end{array}$} & \multirow[b]{2}{*}{$\left|\begin{array}{c}\text { Error in } \\
\omega^{2} \\
\left(\operatorname{rad}^{2} s^{-2}\right)\end{array}\right|$} & \multicolumn{5}{|c|}{$\mathrm{h}_{\mathrm{d}}(\mathrm{cm}) \pm 0.1 \mathrm{~cm}$} & \multirow[b]{2}{*}{$\mathrm{h}_{\mathrm{d}}$ average } & \multirow[b]{2}{*}{$\begin{array}{l}\text { error in } \\
\mathrm{h}_{\mathrm{d}}(\mathrm{cm})\end{array}$} \\
\hline & & & & & & $\mathrm{h}_{\mathrm{d}} \mathrm{l}$ & $\mathrm{h}_{\mathrm{d} 2} 2$ & $\mathrm{~h}_{\mathrm{d}} 3$ & $\mathrm{~h}_{\mathrm{d}} 4$ & $\mathrm{~h}_{\mathrm{d}} 5$ & & \\
\hline 1 & 0.77 & 8.2 & 0.0 & 70 & 0 & 0.6 & 0.7 & 0.5 & 0.5 & 0.8 & 0.6 & 0.2 \\
\hline 2 & 0.53 & 12 & 0.6 & 140 & 15 & 0.9 & 0.9 & 0.8 & 1.0 & 0.9 & 0.9 & 0.1 \\
\hline 3 & 0.36 & 18 & 0.6 & 310 & 22 & 1.6 & 1.6 & 1.6 & 1.5 & 1.5 & 1.6 & 0.1 \\
\hline 4 & 0.28 & 22 & 0.3 & 500 & 14 & 2.8 & 2.8 & 2.8 & 2.8 & 2.8 & 2.8 & 0.0 \\
\hline 5 & 0.21 & 30 & 0.3 & 910 & 19 & 4.5 & 4.3 & 4.5 & 4.3 & 4.3 & 4.4 & 0.1 \\
\hline 6 & 0.19 & 33 & 0.6 & 1100 & 42 & 6.1 & 6.2 & 6.2 & 6.2 & 6.1 & 6.2 & 0.1 \\
\hline 7 & 0.16 & 40 & 1.3 & 1600 & 99 & $\mathrm{~N} / \mathrm{A}$ & $\mathrm{N} / \mathrm{A}$ & $\mathrm{N} / \mathrm{A}$ & $\mathrm{N} / \mathrm{A}$ & $\mathrm{N} / \mathrm{A}$ & N/A & N/A \\
\hline
\end{tabular}

\section{Table 2 - Processed Data calculated using data from Table 1}

Table 1 show cases all data that was measured and further calculations are based on this data only.

Reasons for "N/A" and yellow highlighted cells are explored later. Time period, angular velocity and $\mathrm{h}_{\mathrm{d}}$ were calculated using (19), (20) and (12) respectively.

\section{Data Analysis}

Firstly, $\omega=8.2 \mathrm{rad} \mathrm{s}^{-1}$ had the greatest value of error in $h_{d}$ and is greater than the instrumental error. This was because the parabola that was formed had negligible depth, making its measurement with a ruler difficult as the ruler's least count is $0.1 \mathrm{~cm}$ and doesn't allow measurement of heights to great accuracy.

$\omega=33 \mathrm{rad} \mathrm{s}^{-1}$ had its minimum of parabola located at the base of the beaker, quantitatively seen by the $\mathrm{h}_{0}$ average being $0.0 \mathrm{~cm}$ (rounded to 1 decimal place) and also seen in figure 8 . 

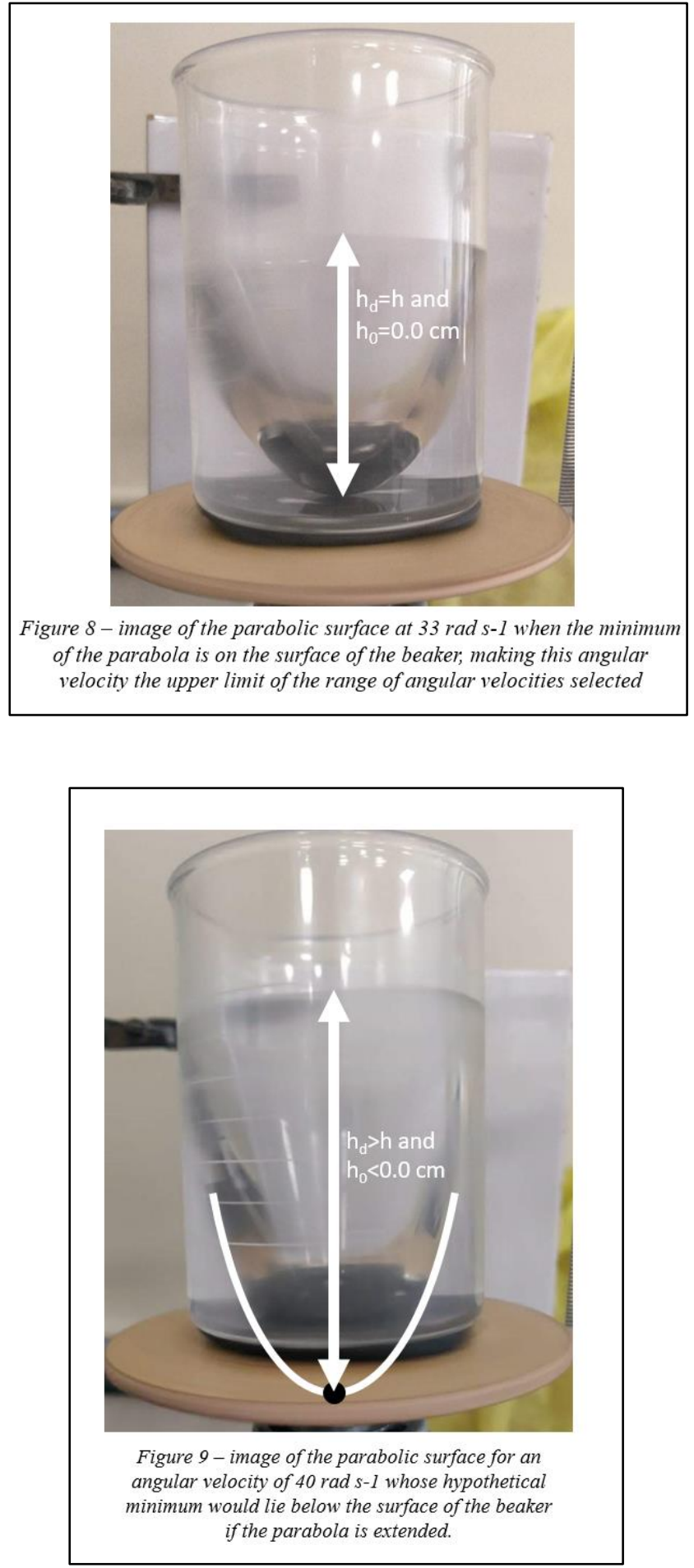

As mentioned already, $\omega=40 \mathrm{rad} \mathrm{s}^{-1}$ was chosen with a value greater than the maximum angular velocity, with a shape shown in Figure 9.

The parabola was extended to visualise a minimum point. Even though the depth of the extended parabola could be determined using image editing software, it would be a non-viable option as it surpasses the conditions of the experiment $\left(h_{d} \leq h\right.$ and $\left.h_{0} \geq 0\right)$. This explains ' $\mathrm{N} / \mathrm{A}$ ' in Table 1 for $\mathrm{h}_{0}$ and $\mathrm{h}_{\mathrm{d}}$. Thereby, the range of angular velocities a limitation of the experiment which can be improved by using a beaker of the same radius but of a larger height such that 
parabolas of greater depths can fit into it, allowing a higher range of independent variables.

The two yellow highlighted cells in Table 1 are two anomalies of the experiment because their values deviate compared to other values of $h_{0}$ and $h$. Thereby, these values were discounted while calculating $h_{d}$, explaining 'N/A' in $h_{d} 1$ and $h_{d} 2$ for the $\omega^{2}=910 \mathrm{rad}^{2} \mathrm{~s}^{-2}$ in Table 3 . This anomaly could exist if the heights were measured before the rotating fluid became stationary with respect to the beaker.

Error in $\mathrm{h}_{\mathrm{d}}$ for $\omega$ (in rad s${ }^{-1}$ ) values of 18 and 22 were low due to the non-negligible parabolic depth that could be measured easily because of low turbulent motion.

As $\omega^{2}$ (in $\mathrm{rad}^{2} \mathrm{~s}^{-2}$ ) increases from 67 towards 1100, $\mathrm{h}_{\mathrm{d}}$ (in $\mathrm{cm}$ ) also increases from 0.6 towards 6.2 (from Table 3 ). The positive correlation between $\omega^{2}$ and $h_{d}$ is as expected from the prediction.

\section{Straight Line Graph Analysis}

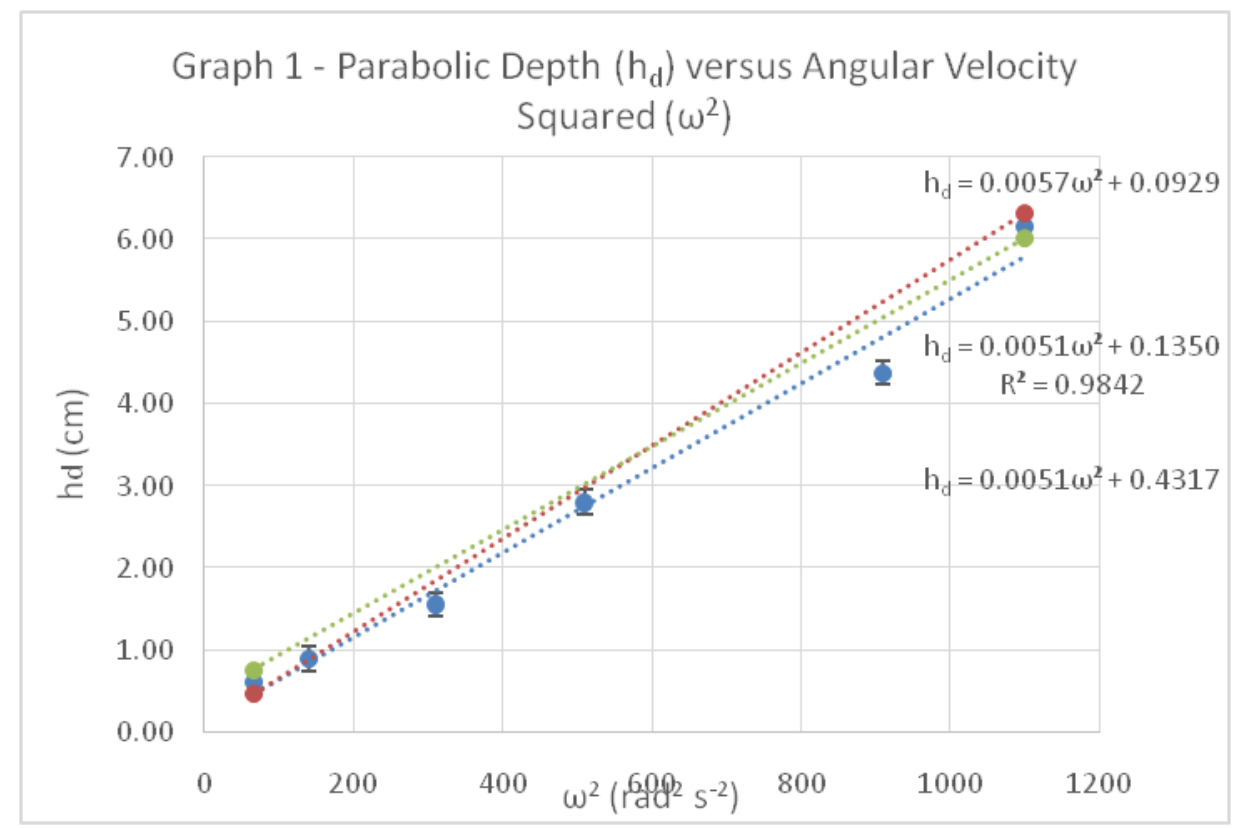

Plotting $\omega^{2}$ versus $h_{d}$ :

The vertical error bar was of a total height of $0.30 \mathrm{~cm}$. The highest error in $\mathrm{h}_{\mathrm{d}}\left(0.15 \mathrm{~cm}\right.$ for $\left.\omega=8.2 \mathrm{rad} \mathrm{s}^{-1}\right)$ was applied to all the other data points as the other error values were too small to be shown.

As predicted, the graph of $\omega^{2}$ versus $h_{d}$ produces a straight line. However, the line doesn't pass through the origin but instead passes through a y-intercept value of 0.135 . Even though systematic errors exist $(=0.135)$ they are negligible and a better methodology will improve the accuracy of the results. A value of $\mathrm{R}^{2}$ close to 1 shows a strong correlation between the two variables and implies that most of the data points can be approximated by the trend line that was created.

It was predicted that the value of the gradient should be:

$$
\text { gradient }=\frac{r_{0}{ }^{2}}{2 g}
$$


The theoretical value is calculated by replacing $\mathrm{r}_{0}=3.25 \mathrm{~cm}$ and $\mathrm{g}=980.67 \mathrm{~cm} \mathrm{~s}^{-2}$ in the above equation:

$$
\begin{aligned}
& \text { gradient }=m_{\text {theoretical }}=\frac{3.25^{2}}{2 \times 980.67} \\
& m_{\text {theoretical }}=0.005385 \approx 0.0054
\end{aligned}
$$

The experimental value of the gradient is:

$$
\begin{gathered}
\text { max gradient }=0.0057 \\
\text { min gradient }=0.0051 \\
\text { error in gradient }=\frac{0.0057-0.0051}{2}=0.0003 \\
m_{\text {experimental }}=0.0053 \pm 0.0003
\end{gathered}
$$

The closeness of the experimental and theoretical values suggests the high accuracy of the data received, implying a strong direct relationship between $\omega^{2}$ and $h_{d}$ and showing that no other variables significantly affected $h_{d}$.

\section{Logarithmic Analysis}

A logarithmic analysis verifies the power to which the angular velocity is related to the parabolic depth. The general positive correlation assumes:

$$
\begin{aligned}
& h_{d} \propto \omega^{n} \\
& h_{d}=K \omega^{n}
\end{aligned}
$$

Taking the natural log on both sides:

$$
\ln h_{d}=\ln \left(K \omega^{n}\right)
$$

Using the properties of logarithms:

$$
\ln \left(h_{d}\right)=\ln (\omega)+\ln (K)
$$

This is synonymous to the equation of a straight line:

$$
y=M x+c
$$

Thereby, plotting $\ln \left(\mathrm{h}_{\mathrm{d}}\right)$ against $\ln (\omega)$ should ideally have $\mathrm{n}=2$ and the value of $\ln (\mathrm{K})$ shows the contribution of the controlled variables. 


\begin{tabular}{|c|c|c|c|c|c|c|c|}
\hline Sr No. & $\begin{array}{c}\omega\left(\mathrm{rad} \mathrm{s}^{-1}\right) \pm \\
0.01 \mathrm{rad} \mathrm{s}^{-1}\end{array}$ & $\begin{array}{c}\mathrm{h}_{\mathrm{d}} \text { average }(\mathrm{cm}) \\
\pm 0.1 \mathrm{~cm}\end{array}$ & $\ln (\omega)$ & $\ln \left(\mathrm{h}_{\mathrm{d}}\right)$ & $\mathrm{h}_{\mathrm{d} m a x}$ & $\mathrm{~h}_{\mathrm{d} m i n}$ & $\begin{array}{c}\text { error in } \\
\ln \left(\mathrm{h}_{\mathrm{d}}\right)\end{array}$ \\
\hline 1 & 8.2 & 0.6 & 2.10 & -0.48 & 0.8 & 0.5 & 0.24 \\
\hline 2 & 12 & 0.9 & 2.48 & -0.11 & 1.0 & 0.8 & 0.11 \\
\hline 3 & 18 & 1.6 & 2.87 & 0.44 & 1.6 & 1.5 & 0.03 \\
\hline 4 & 23 & 2.8 & 3.12 & 1.03 & 2.8 & 2.8 & 0.00 \\
\hline 5 & 30 & 4.4 & 3.41 & 1.48 & 4.5 & 4.3 & 0.02 \\
\hline 6 & 33 & 6.2 & 3.51 & 1.82 & 6.2 & 6.1 & 0.01 \\
\hline 7 & 40 & \multicolumn{7}{|c|}{ N/A } & 3.68 & N/A & N/A & N/A & N/A \\
\hline \multicolumn{7}{|c|}{ Table 4: Data for logarithmic analysis } \\
\hline
\end{tabular}

Here again, "N/A" is written for $\omega=40 \mathrm{rad} \mathrm{s}^{-1}$ as its $\mathrm{h}_{\mathrm{d}}$ values are not considered.

$$
\text { error in } \ln \left(h_{d}\right)=\frac{\ln h_{d} \max -\ln h_{d} \min }{2}
$$

\section{Sample Calculation}

Sample calculation for $\omega=8.2 \mathrm{rad} \mathrm{s}^{-1}$ :

$$
\text { error in } \ln \left(h_{d}\right)=\frac{\ln (0.8)-\ln (0.5)}{2}=0.23500 \approx 0.24
$$

Similar calculations were conducted for other values of $\omega$.

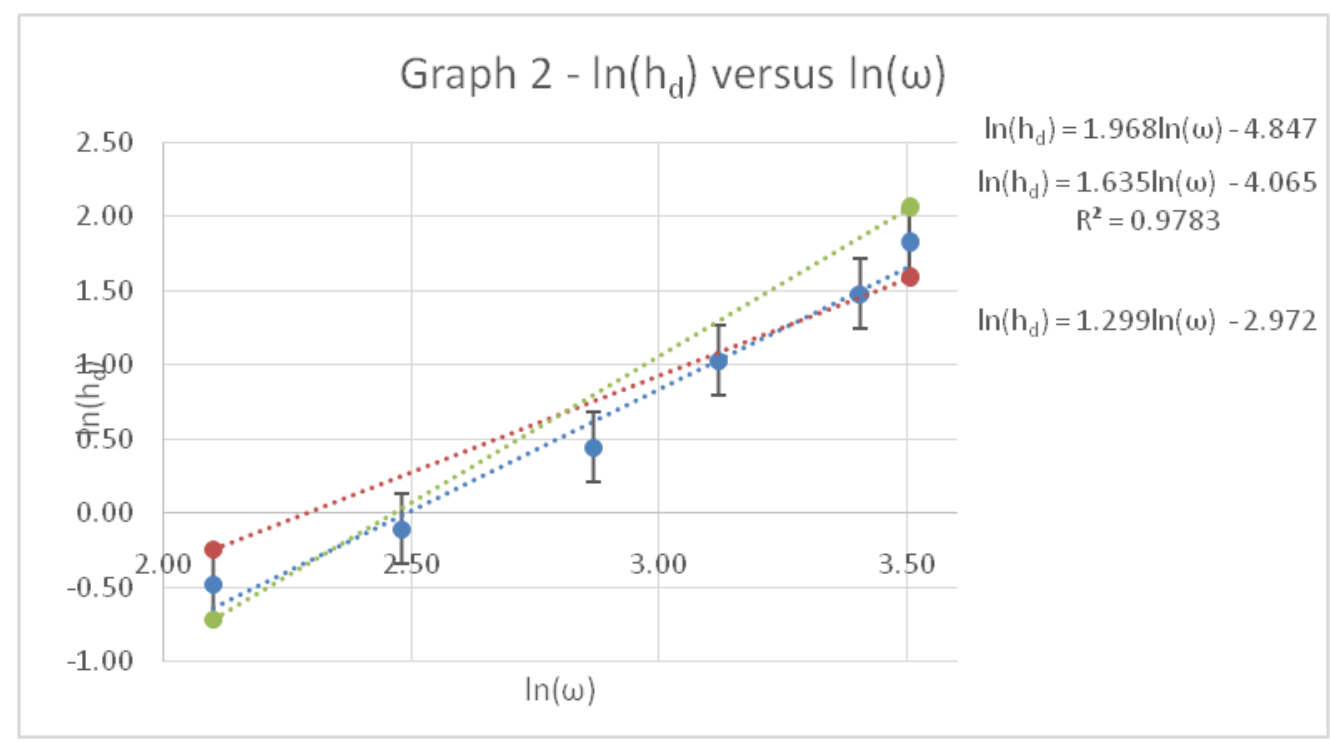

Plotting $\ln \left(\mathrm{h}_{\mathrm{d}}\right)$ against $\ln (\omega)$ :

The equation of the best fit line is:

$$
\ln \left(h_{d}\right)=1.635 \ln (\omega)-4.065
$$

The gradient is 1.64 when rounded to 3 significant figures. 


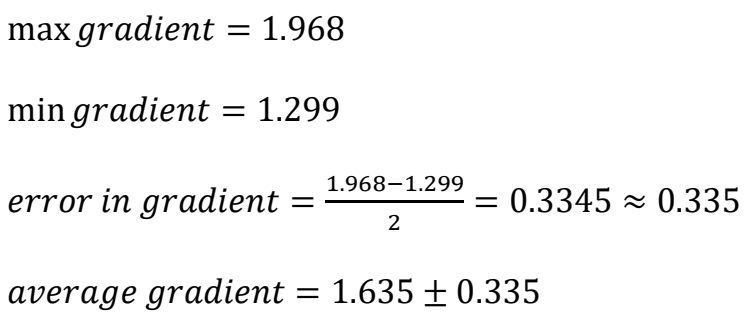

The upper bound for the value accurately predicts the quadratic relationship. The gradient is $1.64 \pm 0.33$ when rounded to 2 decimal places and this estimates the value of $n$ in (23). The y-intercept is:

$$
\begin{aligned}
& c=\ln (K)=-4.065 \\
& K=e^{-4.065}=0.017
\end{aligned}
$$

0.017 is the constant which represents the factor that the controlled variables contribute to influence the parabolic depth.

The logarithmic analysis suggests: $h_{d}=0.017 \omega^{1.64}$ which is an $18 \%$ deviation from $\omega^{2}$.

\section{Evaluation}

Some of the reasons which can explain the deviation above are listed in this section, suggested improvements are also listed.

The human reaction error influences the value of time period to determine the angular velocity. This was tackled by video recording the stopwatch along with the rotating beaker. This allowed the accurate determination of the number of rotations in a 10.00 s period. This can further be improvised by using a photocell connected to a computer.

The point in time at which the height measurements were taken is subjective as enough time had to be given for the rotating water to be stationary with respect to the rotating beaker. The subjectivity in the determination of this point includes inevitable errors. And the early measurement of the depth can lead to misleading values (cells highlighted in yellow in Table 1).

Additionally, difficulties to measure the heights increased when motion got turbulent at higher angular velocities as the beaker began to wobble. This causes a random error seen at $\omega^{2}=910 \mathrm{rad}^{2} \mathrm{~s}^{-2}$, which is away from the trend line in Graph 1, this reduces the precision of the results.

The angular velocity was kept the same by keeping a constant input voltage. However, during the process of data collection, the input voltage, instantaneously, had minor fluctuations (for example from $1.1 \mathrm{~V}$ to $1.2 \mathrm{~V}$ and back to $1.1 \mathrm{~V}$ ). These fluctuations along with resistance in wires can cause negligible changes in angular velocity which directly impacts the parabolic depth. The suggested improvement can be to use a variable speed motor.

A general improvement to correct the errors above is to take more repeats to reduce the errors' effect on the precision of the results.

The y-intercept in Graph 1 is the value of the systematic errors since these errors cause a fixed deviation of the results and cause the line to pass through an intercept rather than the origin (as expected in the prediction assuming a 
perfect experiment). The general method to improve these errors is to utilise a more sophisticated setup that tends to be difficult, expensive and cumbersome to setup in a school laboratory. ${ }^{\text {xvii,xix }}$

\section{CONCLUSIONS}

In conclusion, by means of this experiment, the relationship between the angular velocity of a fluid in rigid body rotation and its parabolic depth was experimentally determined. The theory suggested a linear relation between $h_{d}$ and $\omega^{2}$, however, experimentally a value of $\omega^{1.64}$ was determined - a $18 \%$ deviation. This deviation is explained by the errors, limitations and assumptions in this experiment which inhibits maximum accuracy and precision of the results. The final relationship received through the logarithmic analysis was: $h_{d}=0.017 \omega^{1.64}$. This was explained through the direct relationship between angular velocity and pressure exerted on fluid and consequently a higher parabolic depth.

The biggest factor which leads to this deviation is the basic choice of apparatus used which, as mentioned already, can be used in any school laboratory. Even though these two values (1.64 and 2) are similar, the difference can be minimized by avoiding a few errors to improve the accuracy and precision of the results achieved.

Even though the RQ is answered, further experiments (amongst others) to study Rotating Fluids can be to study:

- Them in a rotating (non-inertial frame) of reference.

- Its applications in parabolic mirrors to determine its focal length.

\section{Endnotes}

1. ' O'Connor, J J, and E F Robertson. "Newton's Bucket." Newton's Bucket, MacTutor History of Mathematics Archive, www-history.mcs.st-and.ac.uk/HistTopics/Newton_bucket.html. [Accessed 10 Dec. 2019].

2. ii Tetlow, Phillip. Understanding Information and Computation: from Einstein to Web Science, by Philip Tetlow, Gower, 2016, pp. 12-13.

3. iii Appleyard, Steve. "Using Spinning Liquids to Simplify the Construction of Parabolic Reflectors in Solar Cookers.”, solarcooking.org/research/SpinningParaboicConcentrators.htm. [Accessed 10 Dec. 2019].

4. iv Wilkes, James $\quad$ O. "Informit." $\quad$ InformIT, 15 Nov. 2017, www.informit.com/articles/article.aspx? $\mathrm{p}=2832417 \&$ seqNum=7. [Accessed 10 Dec. 2019].

5. " Yan, Zixiang, et al. "The Profile of an Oil-Water Interface in a Spin-up Rotating Cylindrical Vessel." American Journal of Physics, vol. 85, no. 4, 16 Jan. 2017, pp. 271-276., doi:10.1119/1.4975125.

6. vi Fitzpatrick, Richard. "Rigid Body Rotation." Rigid Body Rotation, 2006, farside.ph.utexas.edu/teaching/301/lectures/node99.html. [Accessed 10 Dec. 2019].

7. vii Robert W. Fox and John W. Mitchell. "3.6 Fluids in Rigid-Body Motion." Fox and McDonald's Introduction to Fluid Mechanics, Wiley, 2019, pp. 144-148.

8. viii Yan, Zixiang, et al. "The Profile of an Oil-Water Interface in a Spin-up Rotating Cylindrical Vessel.” American Journal of Physics, vol. 85, no. 4, 16 Jan. 2017, pp. 271-276., doi:10.1119/1.4975125. 
9. ix Z. Šabatka and L. Dvořák, "Simple verification of the parabolic shape of a rotating liquid and a boat on its surface," Phys. Educ. 45, 462-468 (Sep. 2010).

10. “ Basu, Swapan. "1.0.0 Introduction to Rheology and Rheological Properties." Plant Flow Measurement and Control Handbook: Fluid, Solid, Slurry and Multiphase Flow, by Swapan Basu, Academic Press, an Imprint of Elsevier, 2019, pp. 615-620.

11. ${ }^{x i}$ Bagley, Mark. "Properties of Matter: Liquids." LiveScience, Purch, 24 July 2014, www.livescience.com/46972liquids.html. [Accessed 19 Dec. 2019].

12. xii C. P. Price, “Teacup physics: Centripetal acceleration,” Phys. Teach. 28, 49-50 (Jan. 1990).

13. xiii Mungan, C.E. "Pressure Exerted by a Rotating Cylinder of Fluid-." United States Naval Academy, U.S. Navy Web Site, www.usna.edu/Users/physics/mungan/_files/documents/Scholarship/PressureRotatingFluid.pdf. [Accessed 10 Dec. 2019].

14. xiv McDonald, Kirk T. "Bernoulli's Equation for a Rotating Fluid." Princeton University: Department of Physics, 2009, www.physics.princeton.edu/ mcdonald/examples/bernoulli_rot.pdf. [Accessed 11 Dec. 2019].

15. ${ }^{\text {xv }}$ Maguire, Megan, and Rachael L Baumann. "Wolfram Demonstrations Project." Pressure of a Rotating Fluid, 17 Jan. 2014, demonstrations.wolfram.com/Pressure of a Rotating Fluid/. [Accessed 9 Jan. 2020].

16. xvi Okiishi, T. H., and W. W. Huebsch. "Fluid Statics." Fundamentals of Fluid Mechanics, by B. R. Munson, Wiley, 2010, pp. 75-77.

17. xvii Wilkes, James O. "Informit." InformIT, 15 Nov. 2017, www.informit.com/articles/article.aspx?p=2832417\&seqNum=7. [Accessed 10 Dec. 2019].

18. ${ }^{\text {xvii }}$ Z. Šabatka and L. Dvořák, "Simple verification of the parabolic shape of a rotating liquid and a boat on its surface," Phys. Educ. 45, 462-468 (Sep. 2010).

19. xix Basta, M, et al. "A Simple Experiment to Study Parabolic Surfaces." Physics Education, vol. 35, no. 2, 2000, pp. 120-123., doi:10.1088/0031-9120/35/2/307.

\section{REFERENCES}

1. Bach, B., et al. "From Newton's Bucket to Rotating Polygons: Experiments on Surface Instabilities in Swirling Flows." Journal of Fluid Mechanics, vol. 759, 2014, pp. 386-403., doi:10.1017/ffm.2014.568.

2. Blokh, M. "Combining Laws of Mechanics and Hydrostatics in Non-Inertial Reference Frames". World Academy of Science, Engineering and Technology, Open Science Index 105, International Journal of Physical and Mathematical Sciences, (2015), 9(9), $513-516$.

3. Borra, Ermanno F. "Liquid Mirrors." Scientific American, vol. 270, no. 2, 1994, doi:10.1038/scientificamerican0294-76.

4. Busse, Friedrich \& Dormy, Emmanuel \& Simitev, Radostin \& Soward, A.M.. (2007). Dynamics of Rotating Fluids. 10.1201/9781420055269.ch3.

5. Grube, Jack. "NOTES: Centripetal Force and Parabolic Surfaces." The Physics Teacher, vol. 11, no. 2, 1973, pp. 109-111., doi:10.1119/1.2349890. 
6. J. M. Goodman, “Paraboloids and vortices in hydrodynamics,” Am. J. Phys. 37, 864-868 (Sep. 1969).

7. Jansson, Thomas R. N., et al. "Polygons on a Rotating Fluid Surface." Physical Review Letters, vol. 96, no. 17, 2006, doi:10.1103/physrevlett.96.174502.

8. Lubarda, Vlado A. "The Shape of a Liquid Surface in a Uniformly Rotating Cylinder in the Presence of Surface Tension." Acta Mechanica, vol. 224, no. 7, 2013, pp. 1365-1382., doi:10.1007/s00707-013-0813-6.

9. Macguire, Shane. "Shane Macguire." Askiitians Discussion Board, AskIITians, 5 June 2015, www.askiitians.com/forums/Mechanics/a-a-fluid-is-rotating-at-constant-angular-veloci_122857.htm. $\quad$ [Accessed $19 \quad$ Dec. 2019].

10. Marshall, John, and R. Alan Plumb. Atmosphere, Ocean And Climate Dynamics. Elsevier Science, 2014, pp. 180-188.

11. McDonald, Kirk T. Rotating Water Bucket With Lid. Princeton University, 2019, http://www.physics.princeton.edu/ mcdonald/examples/bucket.pdf. [Accessed 21 Aug. 2019].

12. Mungan, C. E., and Trevor C. Lipscombe. "Newton's Rotating Water Bucket: A Simple Model." United States Naval Academy, www.usna.edu/Users/physics/mungan/_files/documents/Publications/WAS6.pdf. [Accessed 10 Dec. 2019].

13. Peressini, Anthony L. The Shape of the Surface of a Rotating Liquid: Applications of Elementary Calculus to Physics. Edc/Umap, 1981.

14. R. E. Berg, “Rotating liquid mirror,” Am. J. Phys. 58, 280-281 (Mar. 1990).

15. Sundström, Andréas, and Tom Adawi. "Measuring g Using a Rotating Liquid Mirror: Enhancing Laboratory Learning." Physics Education, vol. 51, no. 5, 2016, p. 053004., doi:10.1088/0031-9120/51/5/053004.

16. Yasuki Nakayama and Robert F. Boucher. "3.4.2 Rotational Motion." Introduction to Fluid Mechanics, ButterworthHeinemann, 2002, pp. 36-37.

17. Pittala, Suresh, and T. Diriba. "Computational Fluid Dynamics Analysis of Impeller Design For A Pump." International Journal of Mechanical Engineering (IJME) 5.4.

18. Poonia, Hemant, and R. C. Chaudhary. "Mass transfer with chemical reaction effects on MHD free convective flow past an accelerated vertical plate embedded in a porous medium." Int. J. of Applied Mathematics \& Statistical Sciences 5 (2016).

19. Umashanker, Hemant Poonia, and S. S. Dhayal. "Unsteady MHD Poiseuille Dissipative Fluid Flow through Porous Medium with Transpiration Cooling." International Journal of Applied Mathematics Statistical Sciences (IJAMSS) 7.4: 59-70.

20. Sekhar, KV Chandra. "MHD free convective heat and mass transfer flow past an accelerated vertical plate through a porous medium with hall current, rotation and soret effects." International Journal of Mechanical and Production Engineering Research and Development 8.3 (2018): 685-706. 